\title{
Direct Carotid Exposure for Neuroendovascular Approaches
}

\author{
Dong Seong Shin ${ }^{1}$ Ali Yilmaz ${ }^{2}$ Ayca Ozkul ${ }^{3}$ Dong Kyu Yeo ${ }^{4}$ Sun-Chul Hwang ${ }^{1}$ Bum-Tae Kim ${ }^{1}$ \\ Address for correspondence Bum-Tae Kim, MD, PhD, Section of \\ Neurointerventional Surgery, Department of Neurosurgery, \\ Soonchunhyang University Bucheon Hospital, Bucheon, Republic of \\ Korea (e-mail: bumtkim@gmail.com).
}

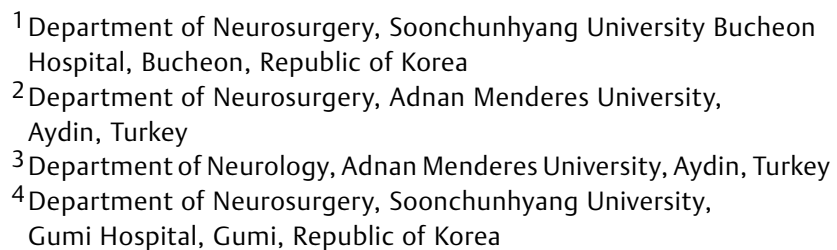

J Neurol Surg A 2016;77:505-510.

\begin{abstract}
Keywords

- direct carotid exposure

- neuroendovascular therapy

- cerebral angiography

Objective The transfemoral approach is a common route for catheterization of the supra-aortic vessels in neuroendovascular therapy. However, in some cases, the patient's anatomy prevents transfemoral catheterization or distal access to the carotid s. In such cases, direct carotid exposure (DCE) for neuroendovascular approaches may be used to treat cerebrovascular diseases.

Methods We present 11 cases in which we were unable to perform the distal approach and DCE was the preferred neuroendovascular treatment procedure.

Results DCE was performed on 11 patients with cerebral aneurysm $(n=8)$, carotid cavernous fistula (CCF) $(n=1)$, malignant brain tumor $(n=1)$, and carotid angioplasty and stenting $(n=1)$. Ten patients were female; one was male. Ages ranged from 63 to 87 years (mean: 71.36 years). Coil embolization was performed on patients with cerebral aneurysm and CCF. The patient with a malignant brain tumor underwent polyvinyl alcohol particle embolization. The only complication was a carotid artery dissection that occurred in one patient during stenting.

Conclusion DCE for neuroendovascular approaches can be used as an alternative for patients with tortuous vasculature access in the femoral route. In such patients, a combination of neuroendovascular treatment and surgery in a hybrid operating room with angiography is preferred.
\end{abstract}

\section{Introduction}

Direct carotid access for the cerebral arteries is an old technique that was described by Moniz in $1927 . .^{1}$ As the development of neuroendovascular treatment approaches proceeded, surgeons began performing direct needle puncture of the common carotid artery (CCA). Vertebral origins and posterior circulation were also evaluated via a subclavian approach. $^{2}$ Transfemoral artery catheterization (TFC) was gradually adopted because it is a less traumatic approach to a site where local pressure can be applied for hemostasis. Moreover, a femoral artery with a larger caliber allows for the introduction of larger sheaths than those in the radial or brachial arteries. Further development of novel devices and techniques during the past 2 decades has allowed to use this access route in most patients. ${ }^{3}$

However, it is sometimes impossible to use the traditional and more familiar transfemoral route in elderly patients characterized by tortuosity of the proximal vasculature and coexistent vasculopathies. In such cases, the neuroendovascular approach received

July 25, 2015

accepted after revision

April 18, 2016

published online

July 1, 2016
C 2016 Georg Thieme Verlag KG Stuttgart · New York
DOI http://dx.doi.org/ 10.1055/s-0036-1584511. ISSN 2193-6315. 
can be performed via radial or brachial routes. Similar to the femoral artery, the brachial and radial arteries are superficial vessels, easy to palpate and compress, and they are not in close proximity to important parenchymal organs. Transradial catheterization can easily be performed in such patients and is commonly used by neurointerventionalists, especially in vertebral artery investigations. However, both arteries (especially the radial artery) are smaller, and intra-arterial local injection of vasodilators is required to minimize vasospasms. In addition, there is the possibility of median nerve injury during transbrachial catheterization (TBC) that clinicians should address. ${ }^{4}$

We present 11 cases in which distal routes could not be used and direct access to the carotid artery was deemed necessary. The CCA was surgically exposed, and catheterization endovascular treatments were performed. Afterward, the CCA was sutured under direct vision.

\section{Material and Methods}

In this study, we investigated 11 patients whose carotid artery was directly accessed during endovascular procedures. Preoperative diagnostic evaluations were performed by contrastenhanced magnetic resonance imaging/magnetic resonance angiography and computed tomography (CT)/computed tomography angiography (CTA). All procedures involved in the endovascular treatment of an intracranial vascular lesion were performed in this manner in a hybrid operating room (OR) equipped with mobile angiography (GE Healthcare, OEC MD 9800, New York, United States) or in an angiographic room (AR) with fixed angiography (Philips Medical, biplane system, Eindhoven, Netherlands) after complete evaluation of the lesions.

Criteria for the direct carotid exposure (DCE) for the neuroendovascular approaches were as follows:

1. Major vessel tortuosity of the thoracic aorta and/or the supra-aortic vessels and bilateral femoral artery stenosis/ occlusion.

2. Inaccessibility of the carotid artery via the radial route so that larger guiding catheters were required for complicated procedures.

3. Medically severely compromised patients who could not tolerate open surgery.
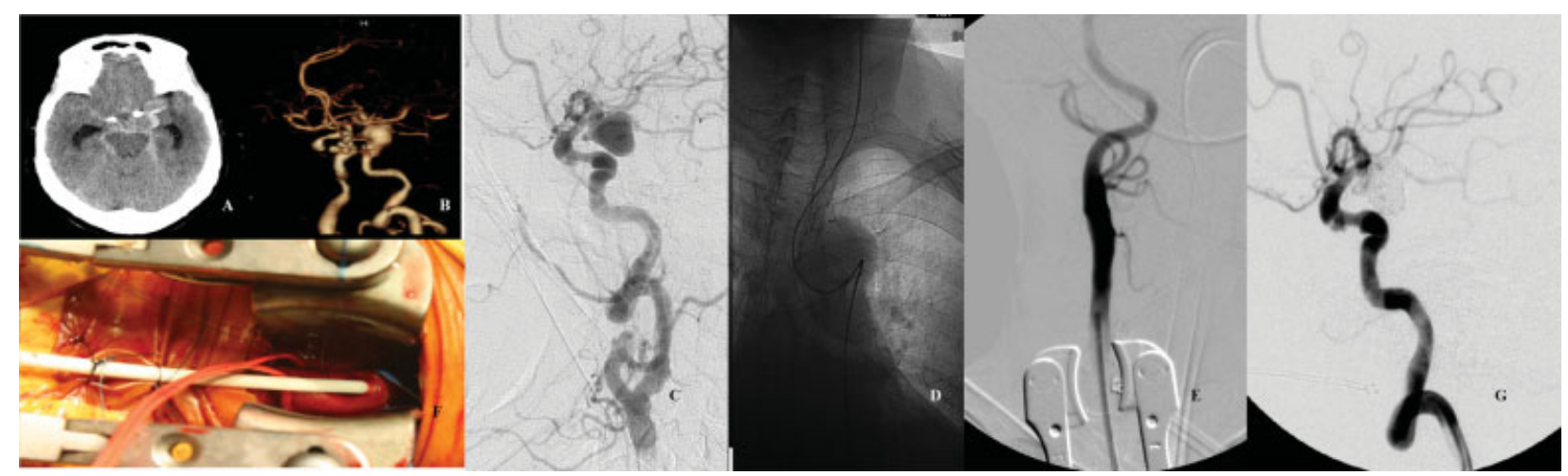

Fig. 1 (A) Diffuse subarachnoid hemorrhage on the basal cisterns and (B) a large posterior communicating artery aneurysm were detected in brain computed tomography and computed tomography angiography in case 1. (C-F) Direct carotid exposure was performed due to severe atherosclerosis and tortuosity. (G) Coil embolization was performed without any complications.
DCE was performed in a hybrid OR or in an angiographic suite under general anesthesia. After general skin drapes, a 4- to 5-cm transverse incision was made along the skin crease of the lower neck. The platysma was cut, and dissection was performed to expose the CCA in the usual manner. Vessel loops were placed on the proximal and distal parts of the exposed CCA, and two 5-0 Prolene anchoring sutures were placed in the central portion. The CCA was punctured with an 18G angiography needle in the area of the anchoring suture, and a 0.035 -inch wire was inserted via fluoroscopy. An introducer sheath was advanced into the vessel over the wire, and the contrast was injected through the ensure vasospasm or dissection. A silk suture was used to anchor the sheath to the skin edge ( $\mathbf{- F i g . ~ 1 F ) . ~ T h e ~ s h e a t h ~ w a s ~ r e m o v e d ~}$ after the neuroendovascular therapy (NET) anchoring sutures were tied tightly. An additional stitch was performed for extra security.

\section{Results}

Eleven patients ranging in age from 63 to 87 years (mean: 71.36 years; one male and 10 females) were examined. Eight patients underwent coil embolization of cerebral aneurysms. The aneurysms were located at the anterior communicating artery (ACoA) $(n=2)$, posterior communicating artery $(\mathrm{PCoA})(n=2)$, or middle cerebral artery (MCA) bifurcation $(n=4)$. One patient with a carotid cavernous fistula (CCF) underwent coil embolization with stent placement. One patient had a malignant tumor at the right temporal lobe with partial polyvinyl alcohol (PVA) embolization. One patient had symptomatic carotid artery stenosis ( $>80 \%$ ) and carotid artery stenting (CAS) with carotid angioplasty. The only complication, a carotid artery dissection, was observed in this patient. During the operations, $6 \mathrm{~F}$ or $7 \mathrm{~F}$ catheters were used. - Table $\mathbf{1}$ summarizes the main observations.

\section{Case Illustrations}

Case 1 was an 80-year-old woman admitted to the emergency department with stupor consciousness. Brain CT and CTA showed a diffuse subarachnoid hemorrhage on the basal cisterns with a large PCoA aneurysm ( - Fig. 1A, B). Catheter angiography performed in the AR showed severe atherosclerosis and sheath to confirm the position of the distal end of the sheath and 
Table 1 Patients who received neuroendovascular treatment via DCE

\begin{tabular}{|l|l|l|l|l|l|l|l|l|l|}
\hline $\begin{array}{l}\text { Case } \\
\text { no. }\end{array}$ & $\begin{array}{l}\text { Age, } \\
\text { gender }\end{array}$ & Diagnosis & Size, mm & $\begin{array}{l}\text { Preoperative } \\
\text { evaluation }\end{array}$ & $\begin{array}{l}\text { Endovascular } \\
\text { procedure }\end{array}$ & $\begin{array}{l}\text { Guide/ } \\
\text { Side }\end{array}$ & Result & $\begin{array}{l}\text { Clinical } \\
\text { outcome }\end{array}$ & Cx \\
\hline 1 & $80, \mathrm{~F}$ & PCoA, RIA & $14.3 \times 16.1$ & AR & OR & $7 F / L$ & NR & MD & None \\
\hline 2 & $68, \mathrm{~F}$ & ACoA, RIA & $4.8 \times 4.0$ & AR & OR & $6 F / R$ & CO & GR & None \\
\hline 3 & $71, \mathrm{~F}$ & MCAB, RIA & $9.2 \times 8.4$ & AR & OR & $6 F / L$ & NR & MD & None \\
\hline 4 & $68, \mathrm{~F}$ & ACoA, RIA & $4.3 \times 2.9$ & AR & AR & $6 F / R$ & CO & MD & None \\
\hline 5 & $71, \mathrm{~F}$ & Brain tumor & $45 \times 40$ & AR & AR & $6 F / R$ & PE & GR & None \\
\hline 6 & $65, \mathrm{~F}$ & Carotid stenosis & $\begin{array}{l}\text { Symptomatic, } \\
80 \%\end{array}$ & AR & OR & $8 \mathrm{~F} / \mathrm{L}$ & CEA failed, & GR & CAD \\
\hline 7 & $63, \mathrm{~F}$ & T-CCF & $10.8 \times 10.4$ & AR & OR & $6 F / R$ & CO & GR & None \\
\hline 8 & $87, \mathrm{~F}$ & PCoA, UIA & $4.9 \times 3.5$ & AR & OR & $6 F / R$ & CO & GR & None \\
\hline 9 & $71, \mathrm{~F}$ & MCAB, RIA & $5.7 \times 5.2$ & AR & OR & $6 F / R$ & CO & GR & None \\
\hline 10 & $74, \mathrm{M}$ & MCAB, RIA & $3.5 \times 1.6$ & AR & AR & $6 F / R$ & CO & GR & None \\
\hline 11 & $67, F$ & MCAB, UIA & $12.1 \times 11.6$ & AR & AR & $7 F / R$ & PO & GR & None \\
\hline
\end{tabular}

Abbreviations: $A C O A$, anterior communicating artery; $A R$, angiography room; CAD, carotid artery dissection; CEA, carotid endarterectomy; CO, complete occlusion; Cx, complication; DCE, direct carotid exposure; GR, good recovery; L, left; MCAB, middle cerebral artery bifurcation; MD, moderate disability; NR, neck remnant; OR, operating room; PCoA, posterior communicating artery; PE, partial embolization; PO, partial occlusion; $R$, right; RIA, ruptured intracranial aneurysm; T-CCF, traumatic carotid cavernous fistula; UIA, unruptured intracranial aneurysm.

tortuosity from the abdominal aorta to the aortic arch and cervical carotid (-Fig. 1C, D). An aneurysm was located on the internal carotid artery (ICA)-PCoA $(14.3 \mathrm{~mm} \times 16.1 \mathrm{~mm})$. The patient was transferred to the OR, and coil embolization with DCE was performed. The aneurysm was occluded with a neck remnant without any complications ( - Fig. 1E-G).

Case 4 was a 68 -year-old woman who was admitted with a severe headache. The CTA showed a saccular aneurysm on the ACoA (-Fig. 2A). The brachiocephalic trunk originated from the proximal aortic arch, and carotid selection failed with all types of angiographic catheters on catheter angiography (-Fig. 2B). DCE resulted in complete occlusion of the ACoA aneurysm with coils performed in the AR with three-dimensional rotational angiography ( - Fig. 2C-E).

Case 11 was a 67 -year-old woman who was incidentally diagnosed with a large right MCA aneurysm on the CTA (-Fig. 3A). The patient had liver cirrhosis, diabetes mellitus, chronic renal disease, and coronary heart disease. Angiogra- phy showed severe atherosclerosis on the thoracic aorta and aortic arch (-Fig. 3B). Carotid selection failed, and the angiogram had to be taken from the contrast injection at the orifice of the innominate artery. The detailed geometry of the aneurysm could not be inferred. According to laboratory findings, the patient had moderate kidney dysfunction (blood urine nitrate $27-30 \mathrm{mg} / \mathrm{dL}$, creatinine $1.5-1.7 \mathrm{mg} /$ $\mathrm{dL}$ ) and a coagulation abnormality due to liver cirrhosis. Physicians recommended the less invasive surgical procedure with restricted use of contrast and careful perioperative care. DCE was performed. The carotid angiogram showed a $12.1 \times 11.6-\mathrm{mm}$ MCA aneurysm with a broad neck ( - Fig. 3C, D). Coil embolization with two microcatheters was performed, and partial occlusion of the aneurysm with preservation of distal arteries was successfully completed with a decreased amount of contrast and reduced operative time (-Fig. 3E). The patient tolerated the operation and recovered well.

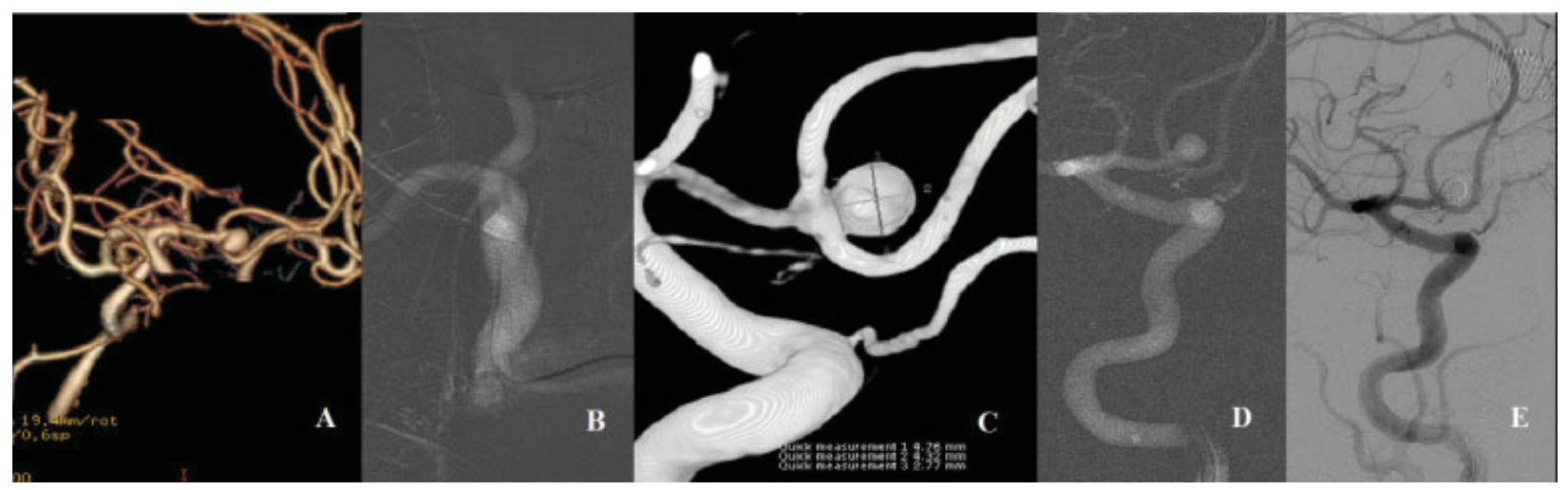

Fig. 2 (A) Brain computed tomography angiography of case 4 revealed a saccular aneurysm on the anterior communicating artery. (B-D) Direct carotid exposure was performed for coil embolization due to tortuosity and a proximally originated brachiocephalic trunk. (E) The aneurysm was completely occluded. 


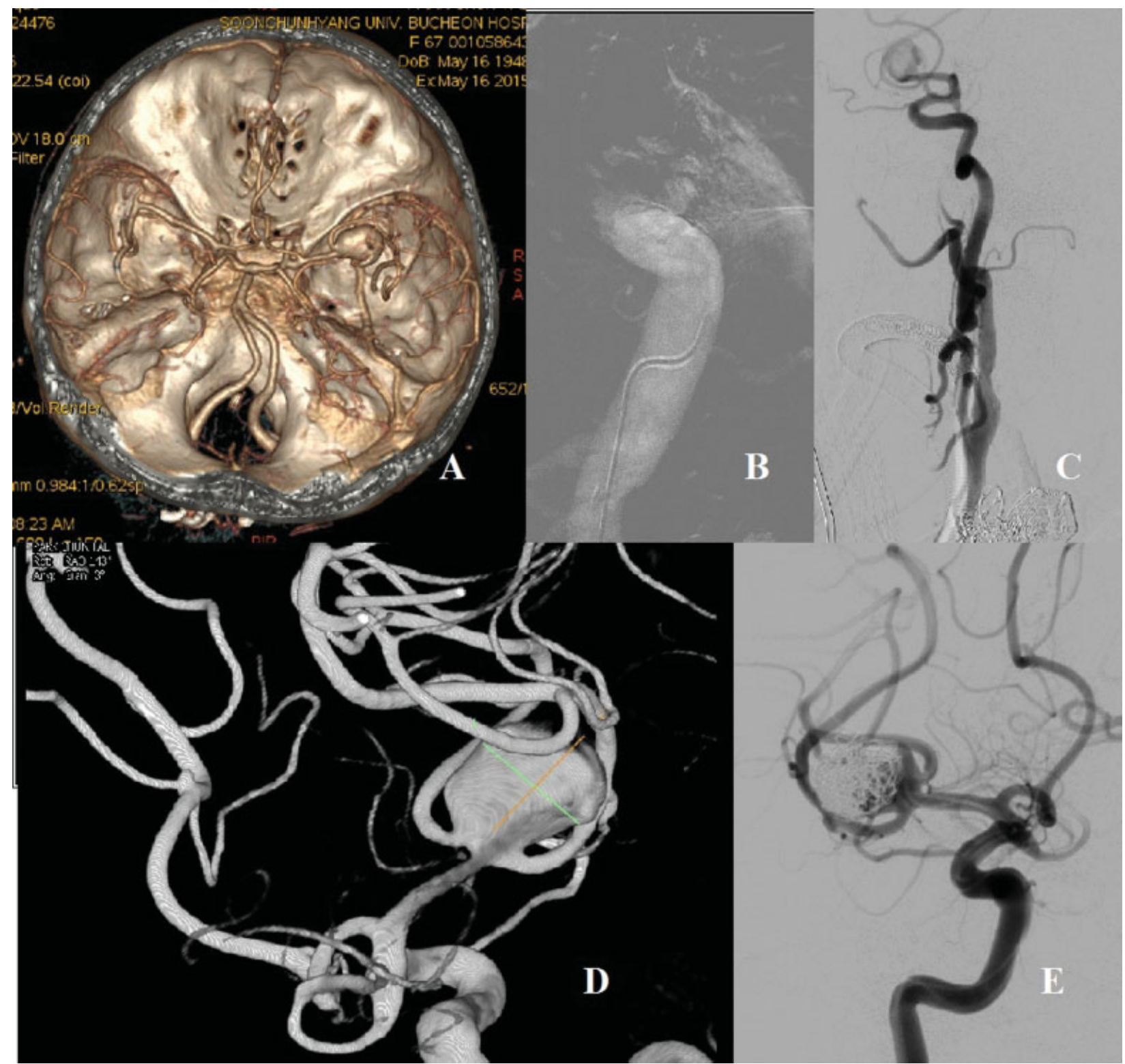

Fig. 3 (A) Brain computed tomography angiography of case 11 revealed a large right middle cerebral artery (MCA) aneurysm. (B) Severe atherosclerosis was detected during angiography, and direct carotid exposure was performed. (C, D) A large MCA aneurysm was partially occluded by coil embolization (E).

\section{Discussion}

Although direct carotid artery access has been used for several years as a common route for cerebral angiography, since the development of newer, more advanced techniques, it has been used only when alternative access is not possible. ${ }^{3-6}$ Today, the primary percutaneous access route for selective catheterization of the carotid arteries is the transfemoral approach. Other alternative vascular access routes include the radial and brachial arteries and percutaneous transcervical CCA access. ${ }^{7}$ However, these routes can be impossible in cases of elongation of the aortic arch, the brachiocephalic trunk, or the carotid artery. Vessel tortuosity and stiffness may limit cranial access and cause a 4 to $6 \%$ failure rate in such procedures. ${ }^{8}$ In addition to anatomical features, other factors, including morbid obesity, severe peripheral atherosclerosis, severe vasculopathies, previous aortic bypass graft surgery, and aortoiliac occlusion, may increase the risk of TFC in $\sim 2$ to $10 \%$ of patients. ${ }^{9,10}$ In such cases, alternative routes should be considered.

Radial and brachial artery routes may also cause difficulties in a tortuous supra-aortic trunk anatomy. Although it is easier to pass through the right carotid and vertebral arteries, ${ }^{3,11}$ the small caliber of the radial artery increases the risk of arterial injury and postoperative arterial occlusion. ${ }^{12}$ In TBC, median nerve injury can be seen following the procedure. ${ }^{13}$ One alternative route is percutaneous CCA access. Difficulties in performing this route include the entry angle of the introducer sheath and difficult access-site management following sheath removal in large guiding catheters, especially in patients taking both antiplatelet and anticoagulant medications. ${ }^{11,14}$ Manual compression or novel closure devices are needed after the 
procedure. ${ }^{7,15,16}$ Additionally the puncture is more distally located and a larger size catheter is usually needed. The main complications seen with this approach include artery dissection and hematomas of the neck that may compromise the airway after the completion of the surgery.

Although DCE for the neuroendovascular approaches is more invasive, it provides safe access because the surgical closure is more efficient than closure devices or manual compression. ${ }^{11}$ It has also been reported that percutaneous hemostatic devices may cause complications such as hematoma, thrombosis, pseudoaneurysm, infection, and arteriovenous fistula. ${ }^{17,18}$ In a recent study, it was determined that bleeding at the puncture site may be a serious problem in cases with extensive perioperative anticoagulation, and it can be controlled more effectively through an open surgical approach than by percutaneous maneuvers. ${ }^{19}$ Therefore, DCE is likely easier, especially for neurosurgeons. DCE can reduce catheter setup time on target ICA and allows easier handling of microdevices and the use of short and soft guidewires because extra stiffness is not necessary. ${ }^{11}$ DCE also offers the advantage of easier and faster catheter exchanges. Therefore, the thromboembolic risk is lower in DCE, which is a familiar procedure for neurosurgeons.

DCE is also recommended in cases of known vascular fragility, such as Marfan disease or Ehlers-Danlos syndrome. In such patients, puncture and repair of the vessel under direct vision is strongly recommended to avoid massive neck hematomas. ${ }^{8,18}$ However, it is important to remember that this surgical procedure may cause cervical hematoma, one of the most frequent local complications. ${ }^{6,20}$ Great care should be taken to avoid bleeding from a back wall puncture. The puncture may be performed with a narrow angle between the catheter course and the artery to reduce the risk of intimal dissection. $^{21}$

DCE can also be performed to treat carotid artery diseases. It was previously demonstrated that CAS with a transcervical approach could be safely performed with good clinical outcomes. In patients at high risk for endarterectomy, retrograde CCA stenting was performed via DCE, and the risk of distal embolization was overcome by clamping the CCA just above the puncture site and aspirating the introducer sheath prior to its removal. This allowed carotid flow reversal and emboli protection without introducing neuroprotective devices. ${ }^{11,15,22}$ The significant athero-occlusive disease of the CCA at the level of cannulation can lead to dissection of the carotid artery. ${ }^{11,22}$ One patient with severe symptomatic ICA stenosis (> 80\%) underwent CAS via DCE. The only complication among all 11 patients was observed in this patient: ICA dissection. After ICA angioplasty, the patient recovered well without any deficits.

In contrast, patients who underwent coil embolization of a cerebral aneurysm, PVA embolization of a malignant brain tumor, and coil embolization with stent replacement in CCF were successfully treated without any complications. None of the patients had neck hematomas, bleeding, thrombosis, or emboli. We believe that intracranial endovascular treatments can be performed more safely than those used to treat extracranial carotid artery diseases. Therefore, in ICA stenosis, the risk of dissection can be determined by evaluating the noninvasive vascular imaging during the preprocedural planning stage. We also believe that combined surgery is more comfortable when a hybrid OR with angiography has been set up, and DCE for neuroendovascular treatment can be safely performed, even in complicated patients with tortuous vascular anatomy.

\section{Conclusion}

There is an urgent need to consider the use of alternative access routes in addition to the transfemoral approach when performing NET, especially in elderly patients. We believe that DCE for neuroendovascular approaches can be used as an alternative in cases with TFC difficulty due to tortuosity and stiffness of the vessels limiting cranial access. Surgical exposure of the cervical carotid artery allows for direct vision of the pathologic vessel and closure through a purse-string suture, which are important advantages when compared with percutaneous CCA puncture.

Financial Disclosure

This work was supported by the Soonchunhyang University Research Fund.

\section{References}

1 Moniz E. L'encéphalographie artérielle, son importance dans la localisation des tumeurs cérébrales. Rev Neurol 1927;2:72-89

2 Diethrich EB, Marx P, Wrasper R, Reid DB. Percutaneous techniques for endoluminal carotid interventions. J Endovasc Surg 1996; 3(2):182-202

3 Jahshan S, Hopkins LN III. If you want to learn new things, read old books: cutdown techniques are well described in the old literature. World Neurosurg 2012;77(1):83-84

4 Yilmaz A, Ozkul A, Shin DS, Im SB, Yoon SM, Kim BT. Morphological assessment of cadaveric radial, brachial and subclavian arteries: a neurointerventional approach. J Korean Neurosurg Soc 2015; 58(6):499-503

5 Guimaraens L, Theron J, Casasco A, Cuellar H. Carotid artery stenting by direct percutaneous puncture. J Vasc Surg 2011; 54(1):249-251

6 Ross IB, Luzardo GD. Direct access to the carotid circulation by cut down for endovascular neuro-interventions. Surg Neurol 2006; 65(2):207-211; discussion 211

7 Larrazabal R, Klurfan P, Sarma D, Gunnarsson T. Surgical exposure of the carotid artery for endovascular interventional procedures. Acta Neurochir (Wien) 2010;152(3):537-544

8 Aletich VA, Debrun GM, Misra M, Charbel F, Ausman JI. The remodeling technique of balloon-assisted Guglielmi detachable coil placement in wide-necked aneurysms: experience at the University of Illinois at Chicago. J Neurosurg 2000;93(3):388-396

9 Levy EI, Kim SH, Bendok BR, Qureshi AI, Guterman LR, Hopkins LN. Transradial stenting of the cervical internal carotid artery: technical case report. Neurosurgery 2003;53(2):448-451; discussion 451-452

10 Yoo BS, Lee SH, Kim JY, et al. A case of transradial carotid stenting in a patient with total occlusion of distal abdominal aorta. Catheter Cardiovasc Interv 2002;56(2):243-245

11 Pipinos II, Bruzoni M, Johanning JM, Matthew Longo G, Lynch TG. Transcervical carotid stenting with flow reversal for neuroprotection: technique, results, advantages, and limitations. Vascular 2006;14(5):245-255 
12 Taha MM, Sakaida H, Asakura F, et al. Access site complications with carotid angioplasty and stenting. Surg Neurol 2007;68(4):431-437

13 Treitl KM, König C, Reiser MF, Treitl M. Complications of transbrachial arterial access for peripheral endovascular interventions. J Endovasc Ther 2015;22(1):63-70

14 Pipinos II, Johanning JM, Pham CN, Soundararajan K, Lynch TG. Transcervical approach with protective flow reversal for carotid angioplasty and stenting. J Endovasc Ther 2005;12(4):446-453

15 Matsumoto H, Fujita K, Miki J, Tsuji N, Terada T, Itakura T. Direct carotid approach for carotid angioplasty and stenting with a small incision: a technical case report. [in Japanese]. No Shinkei Geka 2004;32(7):735-740

16 Perez-Arjona EA, DelProsto Z, Fessler RD. Direct percutaneous carotid artery stenting with distal protection: technical case report. Neurol Res 2004;26(3):338-341

17 Blanc R, Mounayer C, Piotin M, Sadik JC, Spelle L, Moret J. Hemostatic closure device after carotid puncture for stent and coil placement in an intracranial aneurysm: technical note. AJNR Am J Neuroradiol 2002;23(6):978-981
18 Carey D, Martin JR, Moore CA, Valentine MC, Nygaard TW. Complications of femoral artery closure devices. Catheter Cardiovasc Interv 2001;52(1):3-7; discussion 8

19 Dorfer C, Standhardt H, Gruber A, Ferraz-Leite H, Knosp E, Bavinzski G. Direct percutaneous puncture approach versus surgical cutdown technique for intracranial neuroendovascular procedures: technical aspects. World Neurosurg 2012;77; (1):192-200

20 Halbach VV, Higashida RT, Dowd CF, Barnwell SL, Hieshima GB. Treatment of carotid-cavernous fistulas associated with EhlersDanlos syndrome. Neurosurgery 1990;26(6):1021-1027

21 Halbach VV, Higashida RT, Hieshima GB, Hardin CW. Direct puncture of the proximally occluded internal carotid artery for treatment of carotid cavernous fistulas. AJNR Am J Neuroradiol 1989;10(1):151-154

22 Matas M, Alvarez B, Ribo M, Molina C, Maeso J, Alvarez-Sabin J. Transcervical carotid stenting with flow reversal protection: experience in high-risk patients. J Vasc Surg 2007;46(1): 49-54 